\title{
Role of staging laparoscopy with peritoneal lavage cytology in the treatment of locally advanced gastric cancer
}

\author{
Satoru Nakagawa, Atsushi Nashimoto, and Hiroshi Yabusaki \\ The Division of Surgery, Niigata Cancer Center Hospital, 2-15-3 Kawagishicho, Niigata 951-8566, Japan
}

\begin{abstract}
Background. More accurate preoperative staging is necessary to determine the treatment strategy for locally advanced gastric cancer. Laparoscopy has been suggested as an appropriate staging modality. The aim of this study was to clarify the role of staging laparoscopy in patients with locally advanced gastric cancer.

Methods. One hundred patients with primary gastric adenocarcinoma underwent laparoscopy with peritoneal lavage cytology. The disease stages determined were compared with those obtained by conventional methods.

Results. The disease stages were corrected after laparoscopy for 47 of the 100 patients $(47 \%)$, with downstaging in $3(3.0 \%)$ and upstaging in $44(44 \%)$. Peritoneal deposits were found in 7 patients with peritoneal dissemination diagnosed by conventional examination. An unsuspected peritoneal deposit was found in 21 of 93 patients $(22.6 \%)$, and unsuspected free cancer cells without deposits were found in 27 of 93 patients $(\mathbf{2 9 . 0} \%)$. Gastrectomy after staging laparoscopy was performed in 39 patients. Laparoscopy showed no peritoneal deposits in any of these patients. Free cancer cells were found in 9 patients $(23.1 \%)$, but 4 of these had peritoneal deposits at operation. $\mathrm{RO}$ resection was performed in 34 of the 39 patients $(87.2 \%)$. Neoadjuvant chemotherapy after staging laparoscopy was performed in 35 patients. All 35 patients underwent gastrectomy, which resulted in $27 \mathrm{R0}$ and $8 \mathrm{R2}$ resections. Of 18 patients with positive cytology at laparoscopy, 11 had no free cancer cells at operation. Neoadjuvant chemotherapy induced downstaging of the disease in 11 of the 18 patients with positive cytology $(61.1 \%)$. Of 26 patients with massive peritoneal deposits, 4 underwent palliative resection because of pyloric stenosis. Twenty-two patients $(22.0 \%)$ were able to avoid unnecessary laparotomy because of the staging laparoscopy.

Conclusion. Staging laparoscopy with peritoneal lavage cytology is a safe, effective tool in patients with locally advanced gastric cancer, especially in patients receiving neoadjuvant chemotherapy.
\end{abstract}

Offprint requests to: S. Nakagawa

Received: July 25, 2006 / Accepted: November 7, 2006
Key words Gastric cancer · Staging laparoscopy · Peritoneal lavage cytology

\section{Introduction}

Selection of the appropriate treatment for patients with gastric cancer requires accurate tumor staging. Conventional imaging techniques often understage the extent of the intraabdominal spread of advanced gastric cancer, which results in a high rate of unnecessary exploratory laparotomy [1]. The clinical staging can be improved by laparoscopy, since this may identify intraabdominal tumor deposits on peritoneal surfaces, which are not detectable by preoperative noninvasive imaging. Patients with peritoneal seeding found at laparoscopy may be spared an exploratory laparotomy, and they are currently the only ones to benefit from diagnostic laparoscopy.

Peritoneal carcinomatosis is the most frequent pattern of metastasis and recurrence in patients with gastric cancer [2]. Presumably, disseminated lesions originate from free cancer cells exfoliated from the cancerinvaded serosa. To detect these free cells, several Japanese institutions have performed washing cytology [3]. Recently the prognostic value of positive cytology findings was confirmed also in the West [4]. But the role of cytology during laparoscopy in advanced gastric cancer is controversial. In previous reports, cytology during laparoscopy provided no additional information compared to laparoscopy findings alone $[5,6]$.

Neoadjuvant chemotherapy is expected to lead to downstaging of the primary tumors that are thought to be unresectable and thus permit higher curability with subsequent surgery. Several studies showed that preoperative chemotherapy induced downstaging of the disease and resulted in a higher curative resection rate for surgically staged unresectable cancer [7-9]. In patients 
with advanced gastric cancer, accurate staging is necessary to decide on preoperative chemotherapy. Recently, the absence of peritoneal deposits at laparoscopy was included in the eligibility criteria for randomized controlled trials of neoadjuvant chemotherapy.

In the present study we examined the role of laparoscopy with peritoneal lavage cytology in accurate preoperative staging, in order to choose the appropriate treatment modalities for patients with locally advanced gastric cancer.

\section{Patients and methods}

Between January 1999 and June 2005, staging laparoscopy was performed in 100 patients with clinical T3 or T4 advanced gastric adenocarcinoma, at the Department of Surgery, Niigata Cancer Center Hospital. The patients were newly diagnosed and had had no prior treatment. The eligibility for staging laparoscopy included macroscopic type 4 or type 2 and type 3 with positive metastasis in regional lymph nodes. The diagnostic assessments included barium meal, endoscopy, abdominal ultrasonography, and computed tomography $(\mathrm{CT})$ as appropriate to identify metastases or local infiltration. The disease stage was reported according to the criteria in the second English edition of the Japanese classification of gastric carcinoma [10]. The absence or presence of residual tumor after the operation was determined by the $\mathrm{R}$ classification, based on International Union Against Cancer (UICC) criteria [11]. Patients who had positive lavage cytology but no macroscopic peritoneal metastasis were regarded as having had an $\mathrm{R} 0$ resection. Written informed consent to participate in the study was obtained from all patients. The patients were followed up at our hospital until December 2005, with follow-up durations of 17 to 2202 days (median, 505 days). Any deaths after staging laparoscopy, including deaths from other causes, were included in the survival analysis.

Laparoscopy was performed under general anesthesia as an independent procedure, or immediately before surgery. The patient was positioned as for an open upper abdominal procedure, and the operating table was repositioned according to the intraabdominal region to be inspected. A small $(2-\mathrm{cm})$ laparotomy incision was made, into which was inserted a $12-\mathrm{mm}$ disposable trocar for the flexible laparoscope superior to the umbilicus. The abdomen was insufflated with carbon dioxide until a pressure of $10-12 \mathrm{mmHg}$ was reached. A $3-\mathrm{mm}$ access needle was then inserted in the right upper quadrant, under visual control, for washing cytology. All four quadrants of the peritoneal cavity were thoroughly inspected for evidence of malignant deposits, but biopsy of suspect metastases was not performed. Peritoneal lavage fluid was taken from the Douglas pouch and/or left subphrenic space.

At the beginning of the study, immediate laparotomy was performed in asymptomatic patients without evidence of peritoneal deposits (P0) after staging laparoscopy. Gastrectomy was performed for those patients who were diagnosed at laparoscopy as having a few peritoneal deposits graded as P1 according to the first English edition of the Japanese classification of gastric carcinoma [12] Starting in 2001, patients eligible for staging laparoscopy underwent neoadjuvant chemotherapy, with a combination of cisplatin along with S-1 (oral fluoropyrimidine agent), given orally, or 5fluorouracil $(\mathrm{Fu}), \mathrm{CPT}-11$ (irinotecan), or paclitaxel given intravenously, with the aim of downstaging the disease after the staging laparoscopy. Patients with extensive peritoneal dissemination, graded as P2-P3 by the Japanese classification of gastric carcinoma [12], were referred for systemic chemotherapy unless symptomatic disease (obstruction and bleeding) required palliative gastrectomy.

After the neoadjuvant chemotherapy was completed, conventional examinations were routinely carried out to assess the clinical response. The treatment response was categorized using the response assessment of chemotherapy and radiotherapy for gastric carcinoma [10]. A complete response (CR) was defined as $100 \%$ regression of the disease. A partial response (PR) was defined as regression of more than $50 \%$ of the tumor and metastatic lymph nodes, as confirmed by barium meal, endoscopy, and CT scans. Progressive disease (PD) was defined as an increase in the tumor mass or metastatic nodes (or both) or the appearance of new lesion(s). Patients not in these groups were considered to have stable disease (no change; NC).

The $\chi^{2}$ test, Fisher's exact probability test, and the Mann-Whitney $U$-test were used to evaluate differences in clinicopathologic features. Survival was estimated using the Kaplan-Meier method, and statistical differences were analyzed using the log-rank test. A $P$ value of less than 0.05 was considered significant.

\section{Results}

\section{Clinicopathologic characteristics of patients}

Staging laparoscopy was performed in 100 patients with T3 or T4 advanced gastric cancer. Laparoscopy was uneventful in all patients, and there were no procedurerelated complications. The patients' clinicopathologic characteristics are shown in Table 1 . There were 65 men and 35 women, with a median age of 62 years (range, $28-83$ years). In $80 \%$ of the patients, the tumors were macroscopic type 3 or 4 . Histologically, undifferentiated 
tumors (poorly differentiated adenocarcinoma, signetring cell carcinoma, and mucinous adenocarcinoma) were predominant $(73 \%)$. On conventional staging, the tumors were clinical stage II in 42 patients, stage IIIA in 31 , stage IIIB in 12 , and stage IV in 15.

\section{Comparison of conventional and laparoscopic staging}

As shown in Table 2, the disease stages were corrected after laparoscopy for 47 of the 100 patients (47\%), with downstaging in 3 (3\%) and upstaging in 44 (44\%). Peritoneal deposits were found in 7 patients with peritoneal dissemination diagnosed by conventional examination. Table 3 presents clinicopathologic factors relevant to unsuspected peritoneal deposits and free cancer cells. An unsuspected peritoneal deposit was found in 21 of

Table 1. Clinicopathologic characteristics of 100 patients

\begin{tabular}{ll}
\hline Sex, M/F & $65 / 35$ \\
Age (years; mean \pm SD) & $61.0 \pm 11.9$ \\
Cancer location: (U/M/L) & $23 / 38 / 39$ \\
Macroscopic type: $(0 / 1 / 2 / 3 / 4)$ & $0 / 0 / 20 / 29 / 51$ \\
Histology & $27 / 73$ \\
$\quad$ (differentiated/undifferentiated) & \\
cT, (cT3/cT4) & $97 / 3$ \\
cN, (cN0/cN1/cN2/cN3) & $46 / 33 / 12 / 9$ \\
cH, (cH0/cH1) & $99 / 1$ \\
cP, (cP0/cP1) & $93 / 7$ \\
cStage, (IA/IB/II/IIIA/IIIB/IV) & $0 / 0 / 42 / 31 / 12 / 15$ \\
\hline
\end{tabular}

U, Upper third of stomach; M, mid-third of stomach; L, lower third of stomach; cT,clinical tumor; cN, clinical lymph node metastasis; $\mathrm{cH}$, clinical liver metastasis; $\mathrm{cP}$, clinical peritoneal disease; cStage, clinical stage
93 patients $(22.6 \%)$, and unsuspected free cancer cells without deposits were found in 27 of 93 patients (29\%). More patients with type 4 tumors than those with type 2 tumors were found to have peritoneal deposits $(P=$ 0.02 ). Patients with type 3 or 4 tumors were more likely to have free cancer cells than those with type 2 tumors ( $P=0.02$ for type 3 and $P=0.001$ for type 4$)$.

\section{Treatment after staging laparoscopy}

Gastrectomy after staging laparoscopy was performed in 39 patients. At laparoscopy, peritoneal deposits were not found in any of the 39 patients, and free cancer cells were found in 9 patients. At operation, 4 of the 9 patients with positive cytology had peritoneal deposits that had not been discovered at laparoscopy. In 34 patients $(87.2 \%)$, R0 resection was performed, and positive cytology was found in $7(20.6 \%)$ of the 34 patients with $\mathrm{R} 0$ resection. Resection for the remaining 5 pa-

Table 2. Comparison of conventional and laparoscopic staging

\begin{tabular}{llrrrrr}
\hline & & \multicolumn{5}{c}{ Laparoscopic staging } \\
\cline { 3 - 7 } $\begin{array}{l}\text { Conventional } \\
\text { staging }\end{array}$ & & IB & II & IIIA & IIIB & IV \\
\hline II & $n=42$ & 3 & 18 & 0 & 0 & 21 \\
IIIA & $n=31$ & 0 & 0 & 13 & 0 & 18 \\
IIIB & $n=12$ & 0 & 0 & 0 & 7 & 5 \\
IV & $n=15$ & 0 & 0 & 0 & 0 & 15 \\
\hline Correction of disease staging & $47 / 100(47 \%)$ \\
Downstaging & $3 / 100(3 \%)$ & & \\
Upstaging & $44 / 100(44 \%)$ & & \\
& &
\end{tabular}

Table 3. Clinicopathologic factors relevant to unsuspected peritoneal metastasis and free cancer cells

\begin{tabular}{lccc}
\hline & $\begin{array}{c}\text { Unsuspected } \\
\text { peritoneal } \\
\text { deposits } \\
(n=21)\end{array}$ & $\begin{array}{c}\text { Unsuspected free } \\
\text { cancer cells without } \\
\text { peritoneal deposits } \\
(n=27)\end{array}$ \\
Clinicopathologic factors & $n=20$ & $1(5.0 \%)$ & 0 \\
\hline Macroscopic type & $n=29$ & $4(13.8 \%)$ & $7(24.1 \%)$ \\
2 & $n=51$ & $16(31.4 \%)$ & $20(39.2 \%)$ \\
3 & $n=23$ & $7(30.4 \%)$ & $4(17.4 \%)$ \\
4 & $n=38$ & $9(23.7 \%)$ & $13(34.2 \%)$ \\
Cancer location & $n=39$ & $5(12.8 \%)$ & $10(25.6 \%)$ \\
U & $n=27$ & $6(22.2 \%)$ & $5(18.5 \%)$ \\
M & $n=73$ & $15(20.5 \%)$ & $22(30.1)$ \\
L & $n=42$ & $8(19.0 \%)$ & $13(31.0 \%)$ \\
Histology & $7(22.6 \%)$ & $12(38.7 \%)$ \\
Differentiated & $4(33.3 \%)$ & $1(8.3 \%)$ \\
Undifferentiated & $n=31$ & $2(13.3 \%)$ & $1(6.7 \%)$ \\
Stage before laparoscopy & $n=12$ & $n=15$ &
\end{tabular}

U, Upper third of stomach; M, mid-third of stomach; L, lower third of stomach 
tients was R2. Neoadjuvant chemotherapy after staging laparoscopy was performed in 35 patients. At laparoscopy, peritoneal deposits were found in 2 of these patients, and free cancer cells were found in 18. The clinical response (PR in 13, NC in 18, PD in 4), as determined by conventional methods, did not correspond to operative curability. All 35 patients underwent gastrectomy without a second staging laparoscopy, which resulted in 27 with an $\mathrm{R} 0$ resection $(77.1 \%)$ and 8 with an R2 (22.9\%). Positive cytology was found in 1 of the 27 patients that received R0 resection $(3.7 \%)$. Peritoneal deposits were found in all 8 patients who received an $\mathrm{R} 2$ resection. Of the 18 patients with positive cytology at staging laparoscopy, 11 had no free cancer cells at operation. Thus, neoadjuvant chemotherapy induced downstaging of the disease in 11 of 18 patients with positive cytology (61.1\%). Of the 26 patients with P2-P3 disease, 4 underwent palliative resection because of pyloric stenosis. Palliative chemotherapy was performed in 22 patients with $\mathrm{P} 2-\mathrm{P} 3$ disease. As a result, 22 of the 100 patients $(22.0 \%)$ were able to avoid unnecessary laparotomy because of the staging laparoscopy. But 4 of the 22 patients who received chemotherapy required palliative gastrectomy at a later stage due to obstruction and bleeding from the tumor.

\section{Patient outcome}

The overall 5-year survival rate of the 100 patients was $33.1 \%$. The overall survival of the 74 patients without or with a few peritoneal deposits (P0-P1) was $42.7 \%$, but none of the patients with P2-P3 disease survived for more than 3 years after treatment (Fig. 1). Figure 2 shows the survival curves of the 39 patients who received immediate gastrectomy decided upon according to the washing cytology status at laparoscopy. The 5year survival rate of patients with positive cytology was significantly worse than that of patients with negative cytology $(P=0.01)$. Of the 9 patients with positive cytology, 6 developed peritoneal carcinomatosis. Of the 27 patients who received curative resection after staging laparoscopy, 10 died of recurrent disease; 4 of these 10 patients had peritoneal carcinomatosis, 3 had hematological recurrence ( 2 in liver, 1 in bone), 1 had lymph node recurrence, and 2 had combined recurrence ( 1 in peritoneal dissemination and liver, 1 in lymph node and liver). Of the 28 patients who received neoadjuvant chemotherapy followed by curative resection, 3 died of recurrent disease ( 2 with recurrence of peritoneal carcinomatosis and 1 with lymph node metastasis at the paraaortic lesion). Four patients that underwent palliative gastrectomy died after a median of 232 days. Of the 22 patients who received palliative chemotherapy, 18 died after a median of 289 days and 4 were alive with disease at 148, 230, 553, and 563 days.

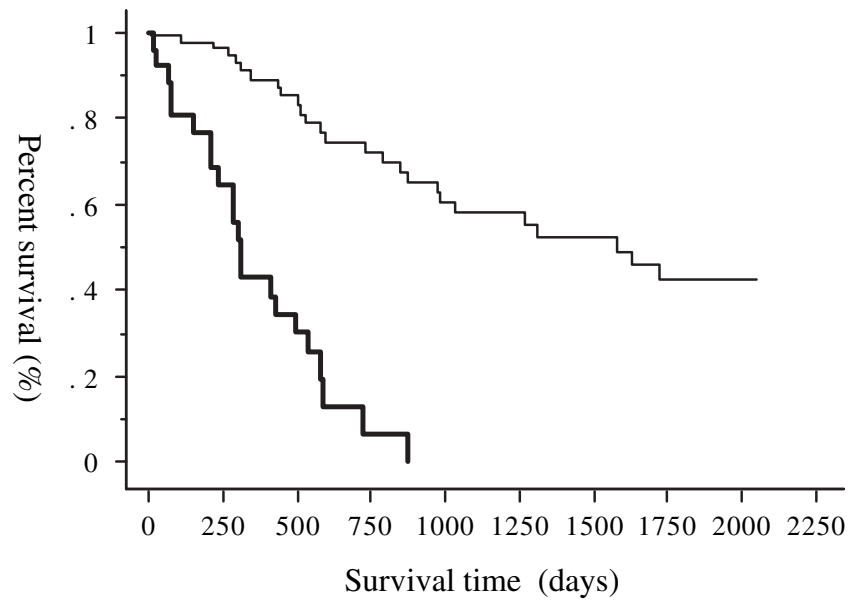

Fig. 1. Survival curves for 100 patients with advanced gastric cancer who received staging laparoscopy. The patients were stratified according to the results of the laparoscopic findings. The difference between the curves was significant $(P<0.0001)$. Thin curve, $\mathrm{P} 0-\mathrm{P} 1$, patients (without or with a few peritoneal deposits; $n=74$ ); thick curve, $\mathrm{P} 2-\mathrm{P} 3$, patients (with extensive peritoneal dissemination; $n=26$ )

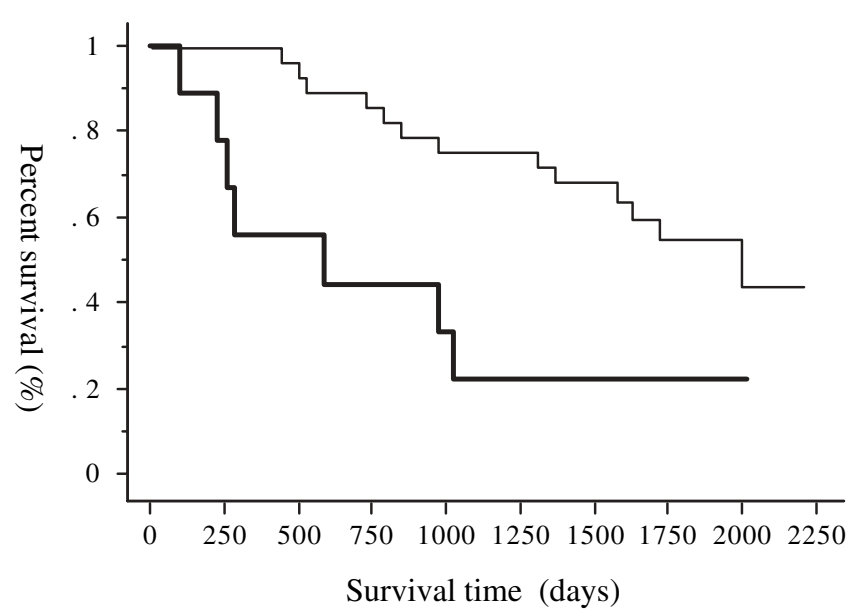

Fig. 2. Survival curves for 39 patients who received immediate gastrectomy according to the status of washing cytology at laparoscopy. The difference between the curves was significant $(P=0.01)$. Thin curve, cytology-negative $(n=30)$; thick curve, cytology-positive $(n=9)$

\section{Discussion}

Laparoscopy has the potential to fulfill two roles in patients with advanced gastric cancers: (1) avoiding an unnecessary laparotomy in patients with incurable metastatic diseases and (2) staging patients for preoperative treatments.

Peritoneal dissemination is the most common pattern of metastasis in patients with incurable neoplastic diseases [2]. Peritoneal metastasis is usually not detected by conventional techniques, but surgical laparoscopy 
offers high accuracy for detecting small intraabdominal metastases [13]. Many laparoscopic studies of patients with gastric cancer have shown that conventional examinations failed to detect peritoneal seeding in about $13 \%$ to $37 \%$ of patients [13-16]. Among the 100 patients enrolled in the present study, 21 (21\%) were found to have unsuspected peritoneal metastasis (a finding which is compatible with the above reports), and these patients were able to avoid unnecessary laparotomy because of the staging laparoscopy.

Early detection of peritoneal dissemination by the cytological examination of peritoneal lavage fluid has been established in Japan [2,3]. The intraoperative cytological examination of peritoneal lavage fluid is important for predicting survival and peritoneal recurrence in patients with gastric cancer. Recently the prognostic value of positive cytology findings was confirmed also in the West [4]. During staging laparoscopy, cytology of peritoneal lavage fluid is easily performed. But the usefulness of cytology during laparoscopy for gastrointestinal malignancies has been a subject of debate in the literature [5,6]. Van Dijkum et al. [5] maintained that cytology of peritoneal lavage fluid should no longer be performed during the laparoscopic staging of gastrointestinal malignancies, because it offered little benefit. However, in their study, patients with gastric cancers were excluded. Sotiropoulos et al. [6] found that cytology during laparoscopy gave no additional information compared to laparoscopic findings alone. In our study, 27 of 93 patients $(29 \%)$ were found to have unsuspected positive cytology without malignant deposits. In 39 patients who received immediate gastrectomy, the survival rate of the patients with positive cytology was significantly worse than the survival rate of those with negative cytology. Also, peritoneal deposits that had not been discovered at laparoscopy were found in 4 patients with positive cytology. These findings reveal that conducting cytology of peritoneal lavage fluid at laparoscopy could be beneficial and could make up for the false-negative results of laparoscopy.

Accurate staging by laparoscopy is necessary in patients with advanced gastric cancers, to assess the benefits of preoperative neoadjuvant chemotherapy. In the past decade, neoadjuvant chemotherapy has attracted interest as a promising treatment strategy [7-9]. Although neoadjuvant chemotherapy has not significantly improved the prognosis for patients with potentially resectable gastric cancers, promising results have come from studies dealing with patients who had surgically staged unresectable cancer. Several studies showed that preoperative chemotherapy induced downstaging of the disease and resulted in a higher curative resection rate for surgically staged unresectable cancer. In the present study, 11 of 18 patients with positive cytology at staging laparoscopy revealed no free cancer cells or downstag- ing at operation. If peritoneal lavage cytology had not been performed at laparoscopy, these patients might have been included in the category of those without negative cytology before treatment. The significance of this change is not clear, but the patients with positive cytology before treatment could have far more advanced diseases relative to those with negative cytology.

The role of a second staging laparoscopy after neoadjuvant chemotherapy is unclear. Conventional imaging examinations have been routinely performed to assess the clinical response to the therapy, but these examinations have not been useful for diagnosing peritoneal metastasis. However, laparoscopy is more invasive and expensive than the conventional examinations. Yano et al. [17] reported that a second staging laparoscopy could accurately assess the response to neoadjuvant chemotherapy to aid in decisions on salvage surgery, especially in patients in whom peritoneal metastasis was the only reason for noncurability. In our study, a second staging laparoscopy was not performed. In 8 of the 35 patients who received neoadjuvant chemotherapy, the resection was $\mathrm{R} 2$. Thus, a second staging laparoscopy could be valuable for these patients to aid in decisions on gastrectomy. The role of a second staging laparoscopy in patients after adjuvant chemotherapy should be examined to determine whether the procedure has additional value for these patients.

In conclusion, staging laparoscopy is a safe, effective tool for diagnosing locally advanced gastric cancer. It can increase the curative resection rate and decrease unnecessary laparotomies in patients with advanced gastric cancer by detecting previously unsuspected peritoneal metastasis. Moreover, cytology of peritoneal lavage fluid at laparoscopy should be done in patients receiving neoadjuvant chemotherapy, as this cytology could be beneficial for these patients.

\section{References}

1. Stell DA, Carter CR, Stewart I, Anderson JR. Prospective comparison of laparoscopy, ultrasonography and computed tomography in the staging of gastric cancer. Br J Surg 1996;83:1260-2.

2. Boku T, Nakane Y, Minoura T, Takada H, Yamamura M, Hioki $\mathrm{K}$, et al. Prognostic significance of serosal invasion and free intraperitoneal cancer cells in gastric cancer. Br J Surg 1990;77:4369.

3. Kodera Y, Yamamura Y, Shimizu Y, Torii A, Hirai T, Yasui K, et al. Peritoneal washing cytology: prognostic value of positive findings in patients with gastric carcinoma undergoing a potentially curative resection. J Surg Oncol 1999;72:60-5.

4. Bonenkamp JJ, Songun I, Hermans J, van de Velde CJH. Prognostic value of positive cytology findings from abdominal washings in patients with gastric cancer. Br J Surg 1996;83: 1672-4.

5. van Dijkum EJM, Sturm PD, de Wit LT, Offerhaus J, Obertop H, Gouma DJ. Cytology of peritoneal lavage performed during 
staging laparoscopy for gastrointestinal malignancies: is it useful? Ann Surg 1998;228:728-33.

6. Sotiropoulos GC, Kaiser GM, Lang H, Treckmann J, Brokalaki EI, Pottgen C, et al. Staging laparoscopy in gastric cancer. Eur J Med Res 2005;10:88-91.

7. Wilke H, Preusser P, Flink U, Gunzer U, Meyer HJ, Meyer J, et al. Preoperative chemotherapy in locally advanced and nonresectable gastric cancer: a phase II study with etoposide, doxorubicin and cisplatin. J Clin Oncol. 1989;7:1318-26.

8. Lowy Am, Mansfield PF, Leach SD, Pazdur R, Dumas P, Ajani JA. Response to neoadjuvant chemotherapy best predicts survival after curative resection of gastric cancer. Ann Surg 1999;229: 303-8.

9. Nashimoto A, Yabusaki H, Tanaka O, Sasaki J, Akiyama N. Neoadjuvant chemotherapy in advanced gastric cancer with noncurative factors: a phase II study with 5-fluorouracil, leucovorin and cisplatin. Gastric Cancer 1999;2:57-63.

10. Japanese Gastric Cancer Association. Japanese classification of gastric carcinoma-2nd English edition. Gastric Cancer 1998;1: $10-24$.
11. Sobin LH, Wittekind CH. TNM classification of malignant tumors. $6^{\text {th }}$ ed. New York: Wiley-Liss; 2002.

12. Japanese Research Society for Gastric Cancer. Japanese classification of gastric carcinoma. Tokyo: Kanehara; 1995.

13. Burke, EC, Karpeh MS, Conlon KC, Brennan MF. Laparoscopy in the management of gastric adenocarcinoma. Ann Surg 1997; 225:262-7.

14. Possik RA, Franco EL Pires DR, Wohnrath DR, Ferreira EB. Sensitivity, specificity and predictive value of laparoscopy for the staging of gastric cancer and the detection of liver metastases. Cancer 1986;58:1-6.

15. Kriplani RA, Kapur BM. Laparoscopy for preoperative staging and assessment of operability in gastric carcinoma. Gastrointest Endosc 1991;37:441-3.

16. Lowy AM, Monsfield PF, Leach SD, Ajani J. Laparoscopic staging for gastric cancer. Surgery 1996;119:611-4.

17. Yano M, Tsujinaka T, Shiozaki H, Inoue M, Sekimoto M, Doki $\mathrm{Y}$, et al. Appraisal of treatment strategy by staging laparoscopy for locally advanced gastric cancer. World J Surg 2000; 24: 1130-6. 